 \\ Iowa Research Online
}

Theses and Dissertations

Summer 2013

\section{Effects of Aging in the Hadronic Forward Calorimeter on the Vector Boson Fusion Higgs Search}

Kamuran Dilsiz

University of Iowa

Copyright 2013 Kamuran Dilsiz

This thesis is available at Iowa Research Online: http://ir.uiowa.edu/etd/4840

\section{Recommended Citation}

Dilsiz, Kamuran. "Effects of Aging in the Hadronic Forward Calorimeter on the Vector Boson Fusion Higgs Search." MS (Master of Science) thesis, University of Iowa, 2013.

http://ir.uiowa.edu/etd/4840.

Follow this and additional works at: http://ir.uiowa.edu/etd

8 Part of the Physics Commons 


\title{
EFFECTS OF AGING IN THE HADRONIC FORWARD CALORIMETER ON THE VECTOR BOSON FUSION HIGGS SEARCH
}

\author{
by \\ Kamuran Dilsiz
}

\begin{abstract}
A thesis submitted in partial fulfillment of the requirements for the Master of Science degree in Physics in the Graduate College of The University of Iowa
\end{abstract}

August 2013

Thesis Supervisor: Associate Professor Jane M. Nachtman 
Copyright by

KAMURAN DILSIZ

2013

All Rights Reserved 
Graduate College

The University of Iowa

Iowa City, Iowa

\section{CERTIFICATE OF APPROVAL}

\section{MASTER'S THESIS}

This is to certify that the Master's thesis of

\section{Kamuran Dilsiz}

has been approved by the Examining Committee for the thesis requirement for the Master of Science degree in Physics at the August 2013 graduation.

Thesis Committee:

Jane M. Nachtman, Thesis Supervisor

Yasar Onel

Edwin Norbeck 
To my family

For their love and support. 


\section{ACKNOWLEDGMENTS}

I would like to express my great appreciation to my supervisor, Prof. Jane Nachtman, for her valuable suggestions and guidance during the development of this work. I also wish to thank her for providing me the knowledge about particle physics.

I would like to greatly thank my thesis committee members: Yasar Onel and Edwin Norbeck. Dr. Onel conveyed me the knowledge about experimental particle physics and the structure of the Detectors. Dr. Norbeck provided me new ideas on the results that I obtained during the study of this work.

I am particularly grateful for the assistance given by Dr. Ping Tan and James W. Wetzel during this study. My special thanks are extended to my friends: Emrah Tiras, Hasan Ogul, Suleyman Durgut and Reddy Pratap Gandrajula.

Finally, I wish to express my love and thankfulness to my family for their support and encouragement in each and every part of my life. 


\begin{abstract}
Vector Boson Fusion (VBF) Higgs production is one of the methods that is being used to search for the existence of the Standard Model (SM) Higgs boson, and the Hadronic Forward Calorimeter (HF) is one of the main calorimeters in the Compact Muon Solenoid (CMS) detector at the Large Hadron Collider (LHC). In this thesis, we looked at the effects of aging in the HF on the VBF Higgs search by using Fast Simulation to model accumulated radiation dosage effects. After proton-proton collisions at the LHC, vector bosons are radiated from quarks and a Higgs boson is produced. In this study, the Higgs boson is forced to decay into tau pairs. The VBF process produces two jets at low angles. We looked for two forward jets, reconstructed in HF and passing analysis thresholds. Thus, we observed jet tagging efficiencies for different levels of radiation damage in HF. Then, we compared the effect of radiation damage for different regions of $\mathrm{HF}$, dividing $\mathrm{HF}$ into four regions. Analysis results showed that the VBF signal is not significantly diminished by the effects of aging in the HF.
\end{abstract}




\section{TABLE OF CONTENTS}

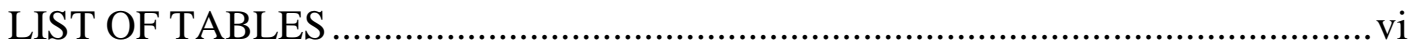

LIST OF FIGURES …………………......................................................

\section{CHAPTER}

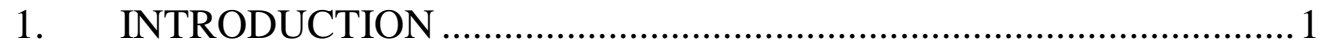

2. THE BRIEF DESCRIPTION OF THE LARGE HADRON COLLIDER AND ITS SUB-DETECTORS .......................................... 3

2.1 Large Hadron Collider ..................................................................

2.2 Compact Muon Solenoid .............................................................

2.3 CMS Hadronic Calorimeter .......................................................... 6

2.4 Hadronic Forward Calorimeter (HF) ............................................



3.1 Radiation Environment and Radiation Damage ...............................

3.2 Radiation Damage Impact on HF ............................................. 10

4. VECTOR BOSON FUSION PRODUCTION AND DI TAU SEARCH FOR THE HIGGS .......................................................................... 13

4.1 Standard Model Higgs Production...................................................13

4.2 Vector Boson Fusion (VBF) Production .........................................14

4.3 Importance of HF to VBF Higgs Search ...................................... 15

5. VBF HIGGS SIGNAL CONSIDERING RADIATION DAMAGE....17

5.1 Searching Radiation Damage Impact on the Jets in HF by Using VBF Signal ..................................................................... 17

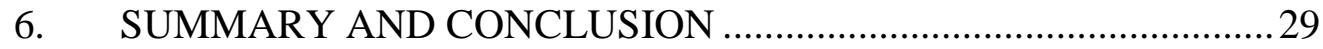

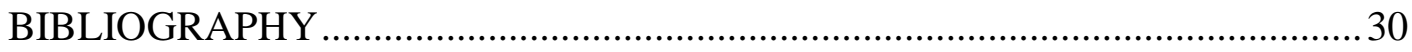




\section{LIST OF TABLES}

Table

3.1 Radiation dosage in three regions of $\mathrm{HF}$ 11

5.1 Number of jets, overall efficiency and statistical errors for all luminosities .....23

5.2 Number of jets, jet tagging efficiencies and statistical errors for all

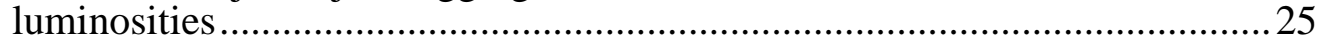




\section{LIST OF FIGURES}

Figure

2.1 A view of the LHC located in a $27 \mathrm{~km}$ circular tunnel................................ 4

2.2 Aerial view of the LHC and its experiments. ..................................... 5

2.3 Compact Muon Solenoid detector ...................................................... 6

2.4 Components of the CMS Hadronic Calorimeter.......................................... 7

2.5 Steel wedges and fibers of the HF during the assembly........................... 8

3.1 Energy recorded in four regions in $\eta$ in the HF, for a $100 \mathrm{GeV}$ input, after $3000 \mathrm{fb}^{-1}$ of integrated luminosity. Region four is closest to the beam.......... 12

4.1 Graphical display of an event recorded with the CMS detector in 2012 at a proton-proton center-of-mass energy of $8 \mathrm{TeV}$ selected as a candidate event by the Higgs search analysis .............................................................. 13

4.2 Initial States for WWH and ZZH Couplings in the VBF Process ................. 15

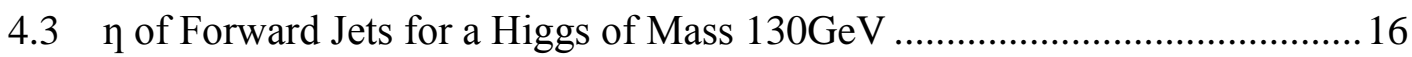

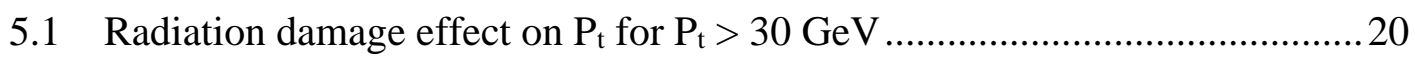

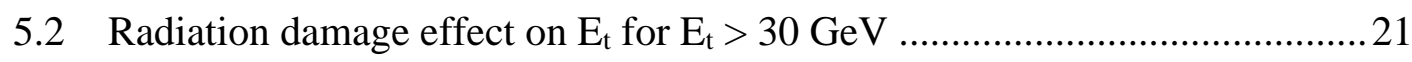

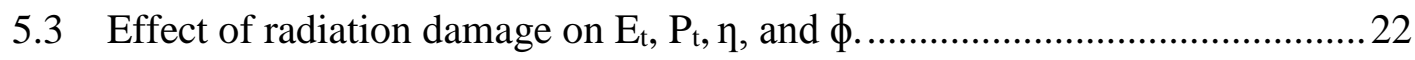

5.4 Overall jet tagging efficiency as a function of integrated luminosity for VBF

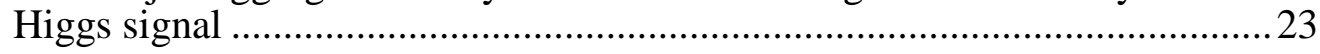

5.5 Jet tagging efficiency as a function of integrated luminosity for four regions

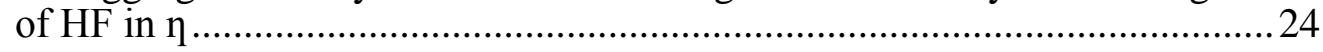

5.6 $\mathrm{P}_{\mathrm{t}}$ distribution for four different regions of HF shown for no radiation

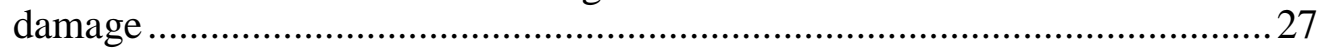

5.7 $\mathrm{E}_{\mathrm{t}}$ distribution for four different regions of HF shown for no radiation damage 


\section{CHAPTER 1}

\section{INTRODUCTION}

The Standard Model (SM) [1] of particle physics is a theory that attempts to explain elementary particles, which make up all matter, and the three forces that govern interactions between these particles. It is the most complete theory that explains subatomic particles. Although the SM finds answers for many physics questions, it is still incomplete. It cannot explain some of the physics questions such as why are there not as many anti-particles as particles in the universe? Where do the fundamental particles get their masses?

In order to solve these problems, Peter Higgs, a professor at the University of Edinburg, introduced a new particle called the Higgs boson [2]. According to the SM, the Higgs boson provides mass to elementary particles; discovery of the Higgs boson is an important step for completeness of the SM. Beyond the SM, other physics theories are also looking for the existence of the Higgs boson. Supersymmetry (SUSY), for instance, proposes the existence of more than one Higgs boson. In contrast, the SM predicts the existence of one Higgs boson.

The latest developments of the CMS and ATLAS experiments show great progress on the search for the Higgs boson. According to their investigations, as announced in March 2013, the new particle observed is the Higgs boson [3]. On the other hand, they are still not sure whether or not this new particle is the SM Higgs boson. In order to be sure about this issue, the CMS and ATLAS collaborations have been looking at properties of the boson.

Vector Boson Fusion (VBF) production is one of the production channels that has been used to study the SM Higgs boson [4]. One of the decay channels used for the Higgs boson is its decay into tau pairs. The tau lepton has a mass of $3500 \mathrm{M}_{\mathrm{e}}$, and its decay mode 
is very fast with a mean lifetime of about $10^{-13}$ seconds $[2,6]$. Due to its lifetime, the channel plays a crucial role. In this analysis, we look at $\tau$ particles decaying into a lepton and two corresponding neutrinos. In the SM, the Higgs boson decay to tau pairs can be examined by the VBF process $\mathrm{qq} \rightarrow \mathrm{qqH}$ considered through the emission of the two jets at high rapidity [5].

VBF production also plays an important role to distinguish Higgs boson signals among the other high transverse momentum $\left(\mathrm{P}_{\mathrm{t}}\right)$ physics events by tagging the forward jets from the interaction [6]. Applying a threshold on the $\mathrm{P}_{\mathrm{t}}$ of the jets allows us to accept or reject the events. This helps to discriminate the VBF Higgs from background events [7]. 


\section{CHAPTER 2}

\section{THE BRIEF DESCRIPTION OF THE LARGE HADRON COLLIDER AND ITS SUB-DETECTORS}

\subsection{Large Hadron Collider}

The Large Hadron Collider (LHC) is the biggest particle physics accelerator built by mankind, and is used to attempt to solve particle physics questions. It is located at the European Organization for Nuclear Research (CERN) in Geneva, Switzerland, and it is in a circular tunnel which is 27 kilometers in length and 175 meters underground [8]. Using the LHC's synchrotron physicists collide two beams of protons, which travel near the speed of light, in opposite directions. Then, they observe the results after these collisions.

The LHC is a tool which allows us to study the forces between elementary particles and the relationship between quantum mechanics and general relativity. Morever, it aims to explain whether the Standard Model is correct or a new model will emerge because of unsatisfactory aspects of the Standard Model.

The current issues that LHC explores are the existence of the Higgs boson, Supersymmetry, Extra Dimensions, and the nature of Dark Matter. First of all, we are still not sure whether or not the Standard Model Higgs boson exists. Physicists are expecting the collider to give an answer about the existence of the Standard Model Higgs boson, and then they will understand how elementary particles get their masses and the Standard Model will be complete. In fact, the announcements by CMS and ATLAS experiments in March 2013 showed that the collider data indicate the existence of the Higgs boson [3]. Currently, they are trying to understand whether this new particle is the SM Higgs boson 
or it is the Higgs boson that is predicted by other physics theories. Second, using the LHC physicists are trying to discover if all particles have Supersymmetric partners. In addition, they are trying to figure out if there are Extra Dimensions in the Universe and explain the nature of Dark Matter. There are three Extra Dimension models. These are Large Extra Dimensions (ADD), Warped Extra Dimensions (RS), and Universal Extra Dimensions (UED) [9]. The ADD model expects missing $\mathrm{E}_{\mathrm{t}}+$ jet signature and the RS model predicts dilepton and dijet resonances [9]. Dark Matter is an unforeseen matter that can only be detected through gravitational interactions. This can be detected while the universe is being pushed by dark energy. Recent Planck results show that the universe is expanding slower than we thought before and there is less dark energy than the previous results [10].

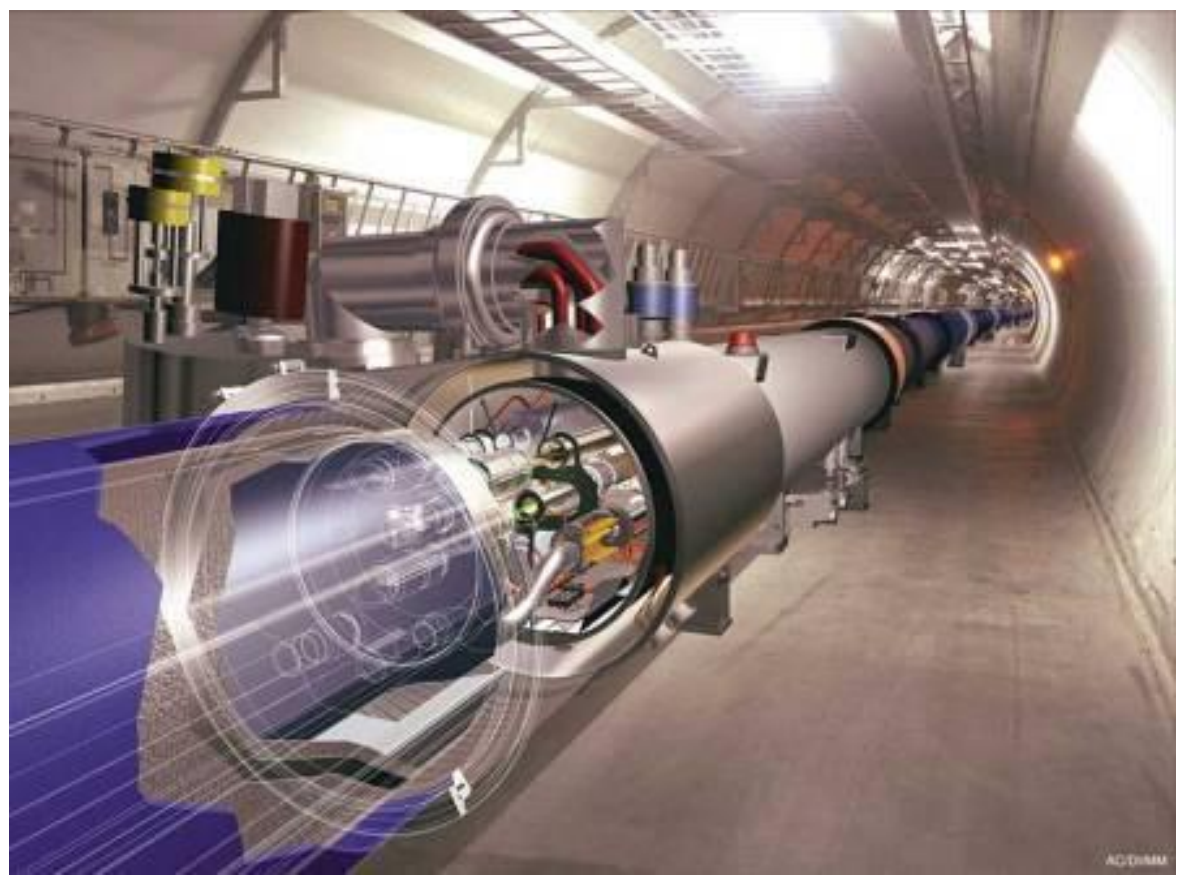

Figure 2.1: A view of the LHC located in a $27 \mathrm{~km}$ circular tunnel. 
There are four large experiments located at the LHC and each of them has different specific goals. These are ATLAS, CMS, ALICE, and LHCb [11, 12]. ATLAS and CMS are the largest and are general purpose detectors. ALICE and LHCb do more specific explorations in heavy ion physics and B physics.

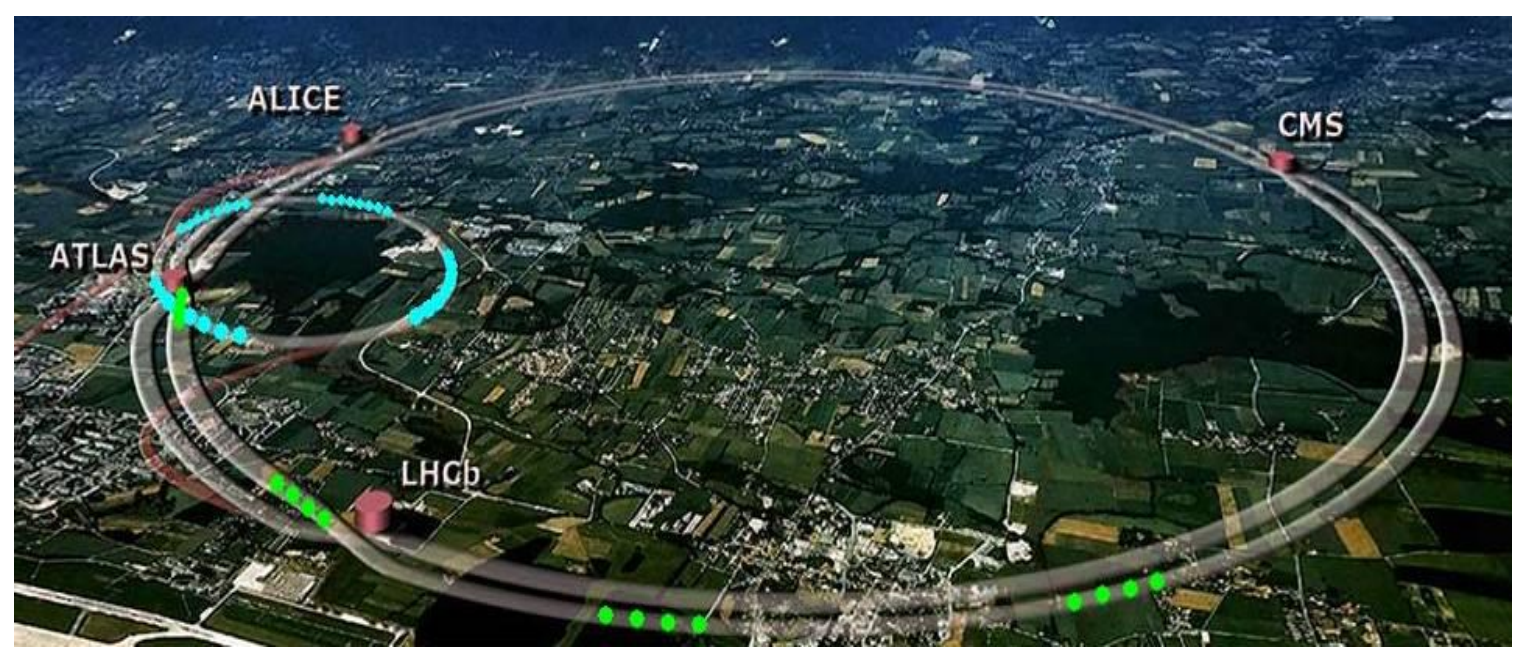

Figure 2.2: Aerial view of the LHC and its experiments.

\subsection{Compact Muon Solenoid}

The Compact Muon Solenoid (CMS) is one of the main particle physics detectors at the LHC. It is 25 meters in length, 15 meters wide, and weighs 12500 tons [11]. It searches for the Higgs boson, Extra Dimensions, and particles that could make up Dark Matter as well as verification of the SM. CMS has a superconducting solenoid that is 13 meters in length and 5.9 meters in diameter [12]. In addition to the solenoid, the CMS detector includes Muon Chambers, Inner Tracker (Silicon Tracker), Electromagnetic Calorimeter, and Hadron Calorimeter.

The CMS software library allows experimenters to do physics analysis. 
Currently, the collection of this software components is called CMSSW; it provides a frame work and data model that allows physicists to do analysis of the data.

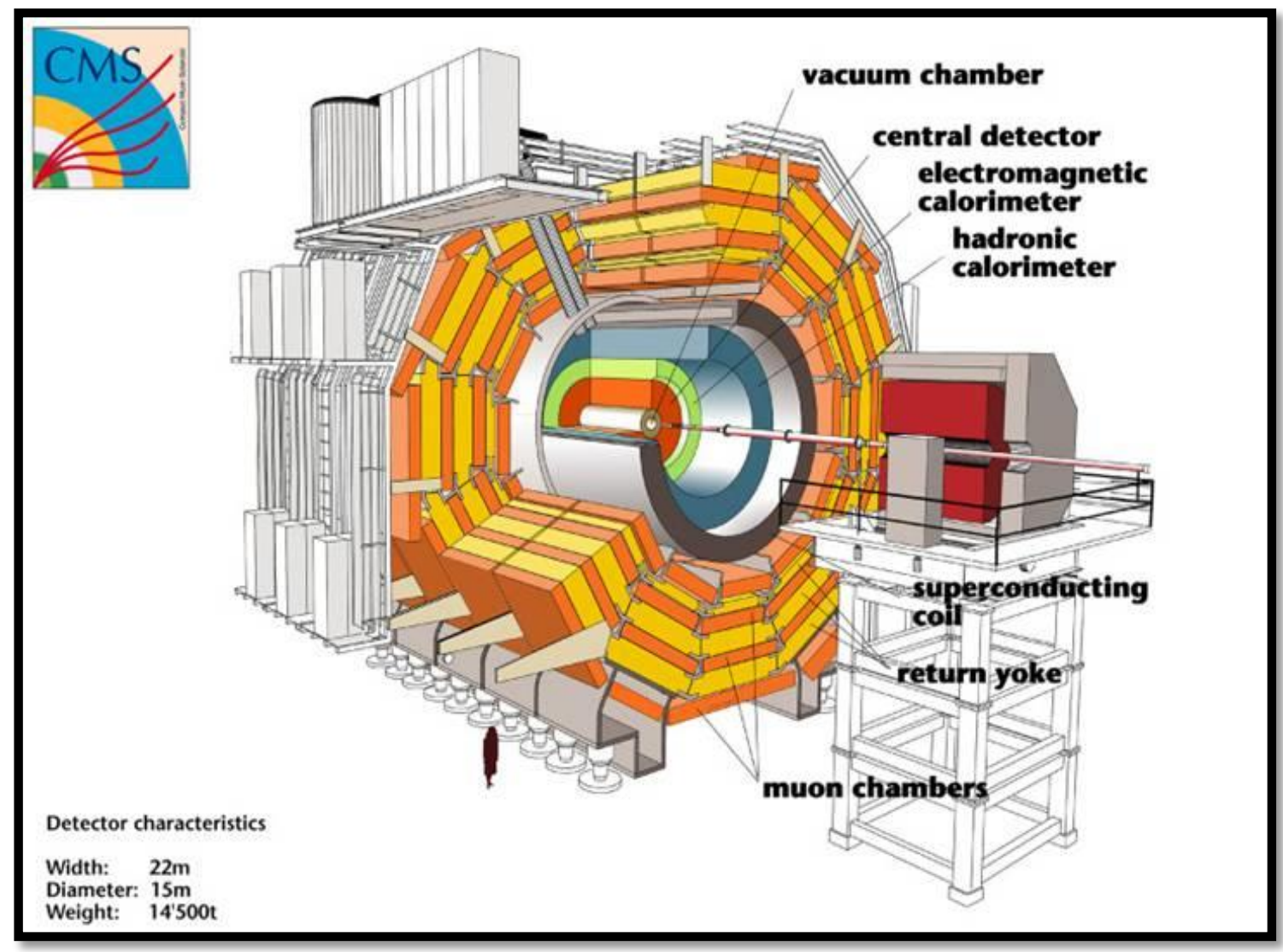

Figure 2.3: Compact Muon Solenoid detector.

\subsection{CMS Hadronic Calorimeter}

The purpose of the Hadron Calorimeter (HCAL) of the CMS detector is to improve the measurement of the missing transverse energy $\left(\mathrm{E}_{\mathrm{t}}^{\text {miss }}\right)$ and measure jet energies. The HCAL is divided into four different calorimeters and each of them covers a different region. These are the Hadronic Forward (HF), Hadronic Barrel (HB), Hadronic Outer (HO), and Hadronic Endcap (HE) calorimeters. Briefly these calorimeters are: 
Hadronic Barrel: It consists of 32 towers and the pseudorapidity range is between $\pm 1.4(-1.4<\eta<1.4)[12]$.

Hadronic Outer: It has scintillators which are $10 \mathrm{~mm}$ in thickness and the pseudorapidity is given by $(-1.26<\eta<1.26)[13]$.

Hadronic Endcap: It has 14 towers and the pseudorapidity range is $(1.3<|\eta|<3.0)$ [12].

Hadronic Forward: It consists of 13 towers and the pseudorapidity range is $(3.0<$ $|\eta|<5.0)[12]$.

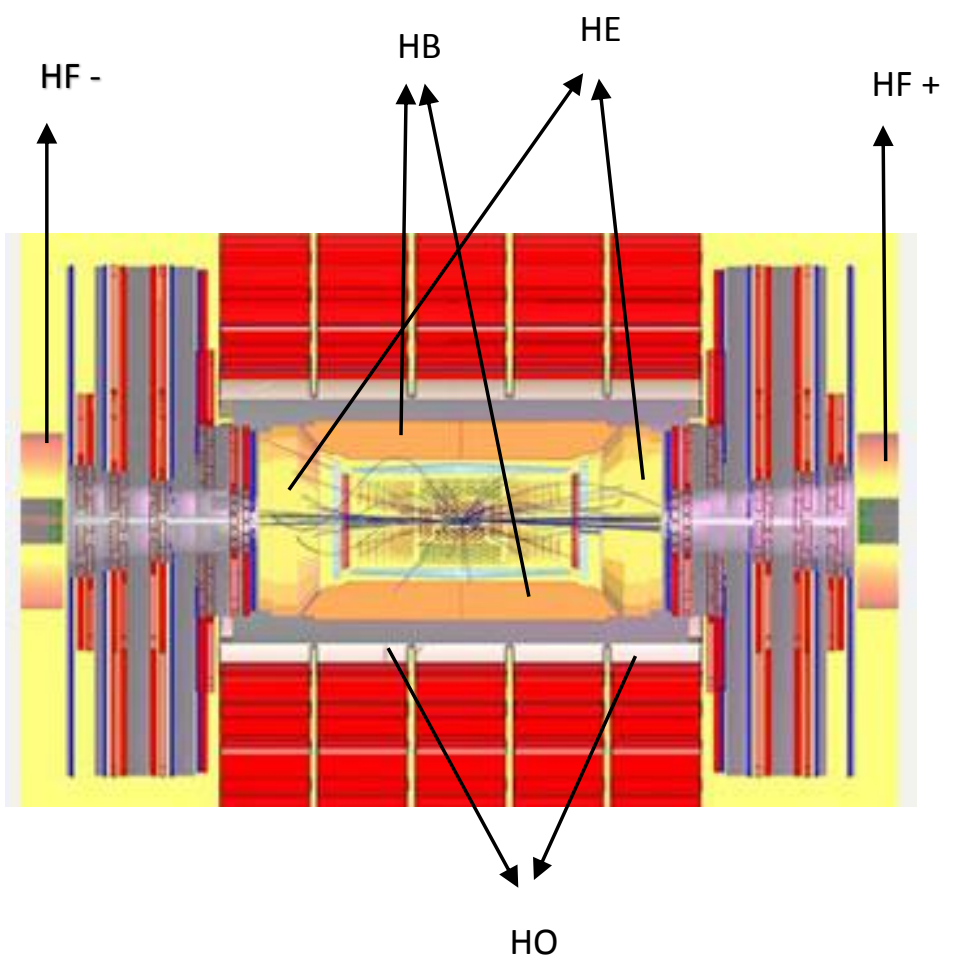

Figure 2.4: Components of the CMS Hadronic Calorimeter.

\subsection{Hadronic Forward Calorimeter (HF)}

The HF is one of the main calorimeters that is located beyond the endcaps of the CMS detector. As can be seen in figure 2.4, there are two HF calorimeters: $\mathrm{HF}+$ and HF-, 
on either side of the interaction point. There is about 11.2 meters distance between each $\mathrm{HF}$ and the interaction point [13]. Each calorimeter is made up of steel wedges making a ring of absorber around the beam line in the forward direction [17]. The HF consists of 13 towers and has a pseudorapidity range ( $\eta$ ) which is $3<|\eta|<5[12]$.

Two types of quartz fibers, long (L) and short (S), are inserted into the steel plates in the HF to detect signals from Cerenkov radiation, which is caused by a charged particle whose velocity is higher than speed of light in the material. The long quartz fibers are 22 $\mathrm{cm}$ longer than the short ones. The purpose of this is to get the range of the data from different shower types. Because long fibers are longer than the short ones, they detect both electromagnetic showers and hadronic showers. In contrast, short fibers only detect the hadronic showers since they do not extend closer to the interaction region. The HF measures the missing transverse energy and jet energies, needed to detect Higgs and SUSY particle production.

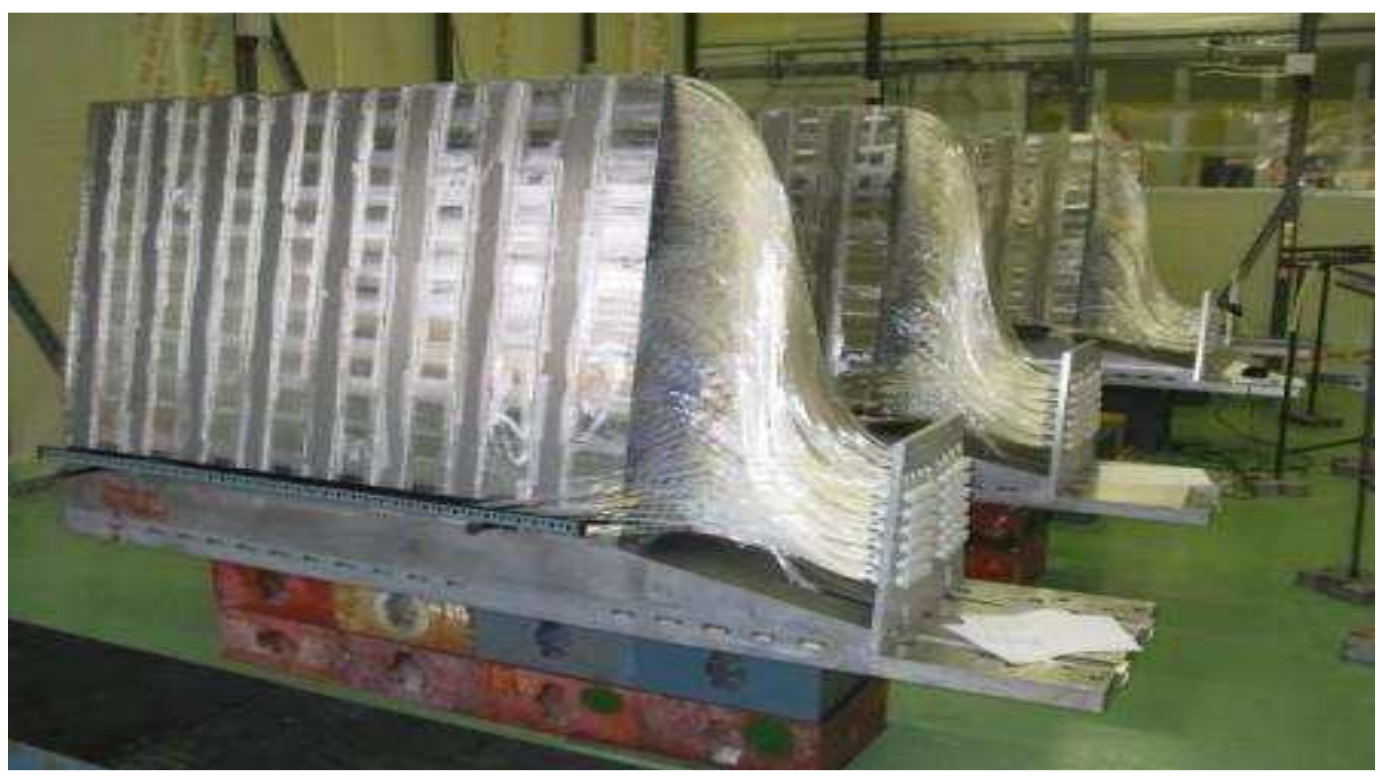

Figure 2.5: Steel wedges and fibers of the HF during the assembly [13]. 


\section{CHAPTER 3}

\section{RADIATION DAMAGE}

\subsection{Radiation Environment and Radiation Damage}

Collisions of proton beams at LHC cause a high radiation environment. General phenomena that cause radiation are beam-induced radiation from proton-proton collisions, beam-gas interactions, and beam halo due to beam interactions in the machine components [14]. However, the radiation of beam-gas and beam-halo interactions are very small compared to the radiation that comes from proton-proton collisions. Due to this, we only consider the radiation from proton-proton collisions in our study. The LHC produces almost $10^{9}$ proton-proton collisions per second at a center of mass energy of $14 \mathrm{TeV}$ while operating at its peak luminosity [15]. These collisions cause energy deposition in the detector. Because of this, assessment of radiation safety and aging of detectors are necessary [15].

The detectors that are close to the beam collision region experience a higher radiation dose and receive high particle fluxes. Because this causes serious damage to the detectors, they are made of radiation hard materials [14]. For instance, the CMS detector is designed to be radiation hard to survive against the radiation environment produced by the proton-proton collisions.

We quantify effects of radiation damage in terms of integrated luminosity because radiation damage is directly related to integrated luminosity, the total number of collisions which have occurred. However, run-time radiation exposure is related to the instantaneous luminosity [15]. 


\subsection{Radiation Damage Impact on HF}

Because the HF is one of the calorimeters that is near the beam collision region of the CMS detector, it is likely to be affected by radiation. Therefore, it is designed to be radiation hard to stand for a long time against the radiation that is derived from protonproton collisions [16]. Computational methods have been developed to investigate the radiation damage impact on $\mathrm{HF}$.

One of the simulation methods used for studies of HF is Fast Simulation, also called FAMOS, Fast Monte Carlo Simulation. The physics processes that are implemented in Fast Simulation are electron Bremsstrahlung, photon conversion, charged particle energy loss by ionization, charged particle multiple scattering, and electron-photon and hadron showering [12].

Within the Fast Simulation is a radiation damage estimate that allows us to understand the performance degradation of the HF detector [16]. The simulation divides HF into one hundred cells in r-z coordinates and each cell is allotted a radiation dose value [16].

Dividing HF into regions is a way to look at the effect of radiation dosage on the calorimeter. The region that is closest to the beam collision will get more radiation dosage than the others. The effect of radiation will decrease respectively from the inner region to the outer one. For example, if the HF was divided into four regions, the inner region would be $(4.5<|\eta|<5.0)$ and the outer region would be $(3.0<|\eta|<3.5)$.

Table 3.1 shows the effect of radiation dosage on three regions of HF for the LHC and SLHC luminosity evolutions. The rings are obtained by dividing HF into three parts. 
The ring that is closest to the beam is Ring 10-13, which corresponds to the pseudorapidity $4.5<|\eta|<5.0$. This ring will receive a higher radiation dose than the others [16].

Table 3.1: Radiation dosage in three regions of HF [16].

\begin{tabular}{|l|l|l|l|}
\hline Luminosity & Ring 1-5 & Ring 6-9 & Ring 10-13 \\
\hline LHC at $10^{34}$ & $1 \mathrm{Mrad} /$ year & $10 \mathrm{Mrad} /$ year & $100 \mathrm{Mrad} /$ year \\
\hline Phase I (at $1.5 \times 10^{34)}$ & $1.5 \mathrm{Mrad} /$ year & $15 \mathrm{Mrad} / \mathrm{year}$ & $150 \mathrm{Mrad} /$ year \\
\hline Phase II (at $\left.3 \times 10^{34}\right)$ & $3 \mathrm{Mrad} /$ year & $30 \mathrm{Mrad} /$ year & $300 \mathrm{Mrad} /$ year \\
\hline SLHC $\left(\right.$ at $\left.10^{35}\right)$ & $10 \mathrm{Mrad} /$ year & $100 \mathrm{Mrad} /$ year & $1 \mathrm{Grad} /$ year \\
\hline
\end{tabular}

The University of Iowa High Energy Group on CMS is using Fast Simulation to study the impact of Radiation Damage on the HF. In order to understand the impact of radiation on the detector, the group used distinct radiation damage fibers for different towers of HF. Putting these fibers between different towers, they transmitted and reflected signals through the fibers to see attenuation of light due to radiation damage [16]. These fibers monitor aging effects in situ. In addition, dedicated studies were performed where fibers of the same type as used in the HF were irradiated, and the attenuation as a function of dosage measured [17]. The relationship between attenuation and dosage was parametrized as $\frac{I(\lambda, D)}{I(\lambda, 0)}=e^{\left(\frac{L}{4.343}\right) \alpha(\lambda)\left(\frac{D}{\mathrm{Ds}}\right)^{\beta(\lambda)}}[16,17]$. This equation is used in the Fast Simulation to estimate the attenuation in each cell in HF. In this formula, I is the intensity, $\lambda$ is wavelength, $\mathrm{D}$ is dosage, $\mathrm{L}$ is luminosity in $\mathrm{fb}^{-1}, \alpha$ and $\beta$ are functions of wavelength and derived from data, and $\mathrm{D}_{\mathrm{s}}$ is a normalization constant. 


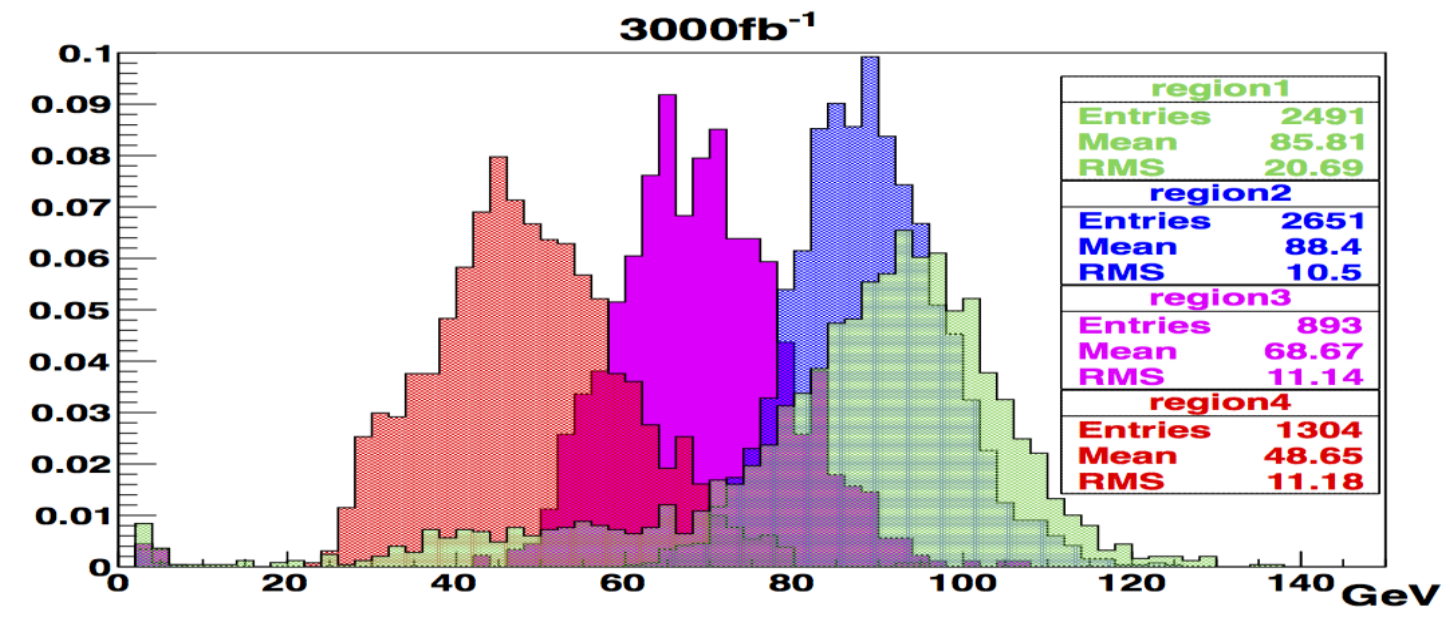

Figure 3.1: Energy recorded in four regions in $\eta$ in the $\mathrm{HF}$, for a $100 \mathrm{GeV}$ input, after $3000 \mathrm{fb}^{-1}$ of integrated luminosity. Region 4 is closest to the beam [16].

Using the Fast Simulation method with radiation damage as a function of luminosity incorporated, we have explored the performance of the HF detector and the impact of radiation damage on HF. We examined how the HF fibers will be affected after being dosed up to $1 \mathrm{Grad}$, using an attenuation model as a function of radiation dosage [16]. Then, we can see how HF performs after taking $3000 \mathrm{fb}^{-1}$ integrated luminosity. The figure 3.1 shows the light attenuation in the HF detector for $3000 \mathrm{fb}^{-1}$ of integrated luminosity [16]. In this figure, the regions correspond to the HF towers and $3000 \mathrm{fb}^{-1}$ corresponds to 10 years of LHC operation. As can be seen in the figure, the most attenuation is in region 4 , which is the highest eta region $(4.5<|\eta|<5.0)$. However, there is less attenuation for the low eta region [16]. Typically the energy recorded in the calorimeter would be calibrated to remove the effects of attenuation. However, this has not been done for our study. 


\section{CHAPTER 4}

\section{VECTOR BOSON FUSION PRODUCTION AND DI TAU SEARCH FOR THE}

\section{HIGGS}

\subsection{Standard Model Higgs Production}

The Standard Model (SM) is a well-accepted particle physics theory that proposes elementary particles and three forces between these particles. This model does not provide a mechanism for particle mass [2]. Then, how do these particles have mass in reality although they do not have mass in the theory?

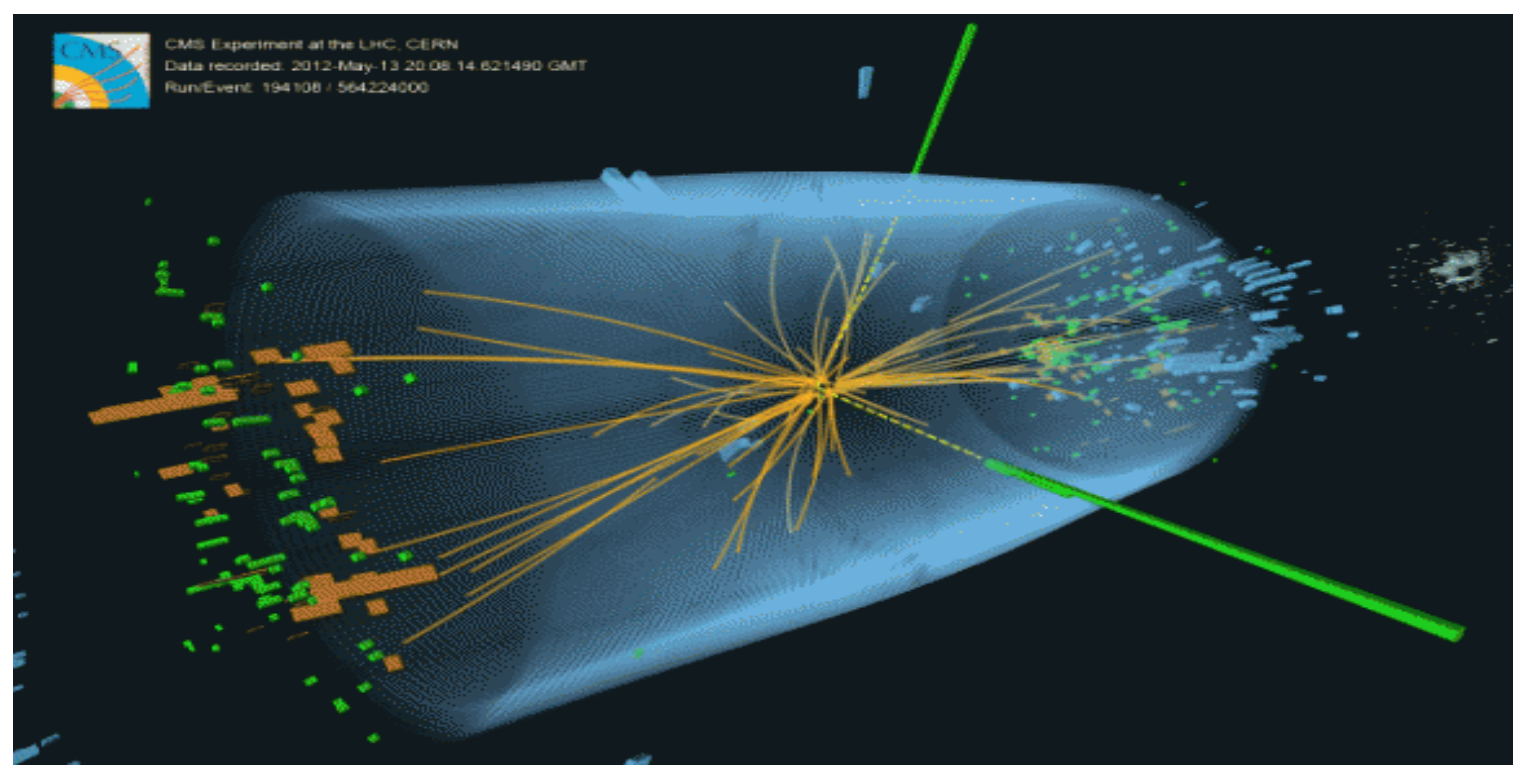

Figure 4.1: Graphical display of an event recorded with the CMS detector in 2012 at a proton-proton center-of-mass energy of $8 \mathrm{TeV}$ selected as a candidate event by the Higgs search analysis [18].

In 1964, Peter Higgs, a professor at the University of Edinburg, introduced a new particle called the Higgs boson and its field called the Higgs Field [2]. He claimed that the 
Higgs field extends throughout the universe and all particles interact with this field, getting their mass from this field through the Higgs mechanism.

Although some theories such as Supersymmetry (SUSY) claim the existence of more than one Higgs boson, the SM predicts the existence of one Higgs boson called the Standard Model Higgs Boson. Until 2013, physicists were not sure whether or not the Higgs boson exists even though theoretically they know its decay process and some properties. However, in March 2013, CMS and ATLAS collaborations reported that they believe that the newly discovered particle is the Higgs boson [3]. In order to test this, they checked the spin and parity of this new particle. Then, the results showed spin zero and positive parity which are predicted for the Higgs boson [3]. Although this result shows that the new particle is a Higgs boson, CMS and ATLAS groups are still need to know what kind of Higgs boson it is. Is it the Standard Model Higgs boson? Or, does it belong to other theories beyond the SM?

In order to solve this mystery, the CMS and ATLAS collaborations study properties such as the decay rate of the Higgs boson to other particles [3]. Decay channels that have being studied for the Higgs boson are fermion- anti-fermion, Higgs decay to weak bosons, and Higgs decays to gluons [19]. In addition to decay channels, there are also the Higgs boson production channels to be considered: these are gluon-gluon fusion, weak boson fusion, and Higgs-strahlung [19].

\subsection{Vector Boson Fusion (VBF) Production}

VBF is one of the production methods that has been used to search for the Higgs boson. This process is the second largest process at LHC [20]. Upon a proton-proton collision, vector bosons are radiated from quarks and a Higgs boson produced at the center. 
The VBF production is expected to be a mode for discovery and study of the Higgs boson at the LHC [4].


Figure 4.2: Initial States for WWH and ZZH Couplings in the VBF Process [21].

Higgs boson decay processes that are being examined by the VBF analysis are $\tau \tau$, WW, and $\gamma \gamma$ decay channels. The CMS collaboration is working on these channels for an integrated luminosity of $30 \mathrm{fb}^{-1}$ using data collected in 2011 and 2012 [21]. The cross section measurement of the VBF process can be used to measure the initial states of Higgs couplings [21].

\subsection{Importance of HF to VBF Higgs Search}

After proton-proton collisions at the LHC, the vector bosons that are radiated by quarks fuse and produce a central high $\mathrm{P}_{\mathrm{t}}$ Higgs and two jets which continue at low angles with respect to the beam. In order to see the importance of HF to the VBF Higgs search, one looks at the two forward jets in the detector, often measured by the HF. These forward jets are used to tag VBF signals; having only two energetic jets allows one to eliminate other background channels.

In order to be sure that these jets are in the forward region $(|\eta|>3.0)$ some requirements need to be satisfied. First of all, jets should be in opposite hemisphers, $\eta_{1} \bullet \eta_{2}$ 
$<0[3,7]$. Then, jets should be well separated in the detector $(\Delta \eta>4.5)[7]$. Last, the transverse momentum $\left(\mathrm{P}_{\mathrm{t}}\right)$ for each jet must be over a certain threshold [7].

Green: $P_{t}>10 G e V$, Red: $P_{t}>20 G e V$, Blue: $P_{t}>30 G e V$

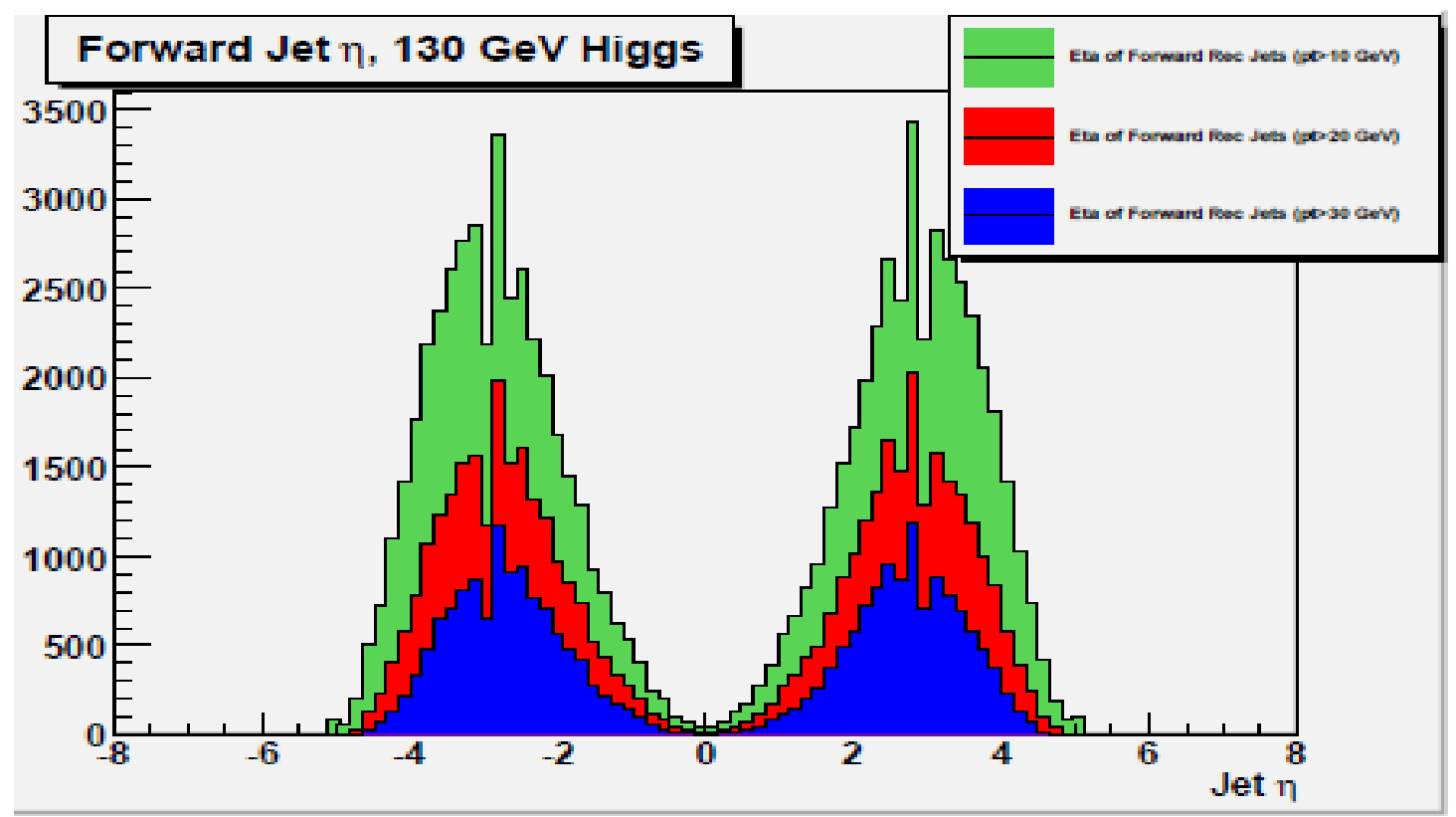

Figure 4.3: $\eta$ of Forward Jets for a Higgs of Mass $130 \mathrm{GeV}$ [7].

Figure 4.3 shows the $\eta$ distribution of jets in the VBF Higgs signal. There is a threshold on these jets at 10,20, and $30 \mathrm{GeV}$ indicated by green, red, and blue respectively. As can be seen in figure 4.3, the number of jets decreases while the threshold momentum increases. A large fraction of the VBF Higgs events have at least one jet in the HF. 


\section{CHAPTER 5}

VBF HIGGS SIGNAL CONSIDERING RADIATION DAMAGE

\subsection{Searching Radiation Damage impact on the Jets in HF by Using VBF Higgs}

\section{Signal}

Using Pythia MC Simulation of the VBF Higgs process with Fast Simulation of the CMS detector including the radiation damage model, we examined the effect of radiation damage on the VBF Higgs signal in the HF calorimeter. We generated 500K events for 0, $20,100,200,500,1000$, and $3000 \mathrm{fb}^{-1}$ integrated luminosity. Then, we analyzed the output files that we got from this process to study the physics impact of radiation damage.

In this analysis we have two types of jets, generated and reconstructed(calo) jets. Generated jets are the jets produced from generator level MC particles. Calo Jets are the jets produced from CMS calorimeter towers.

We started our analysis by matching both of our jets in the HF by minimizing the difference in $\eta$ and $\phi$ between the generated and reconstructed jets. We describe this requirement by formula (1). After matching these two jets, we applied a threshold on $\mathrm{P}_{t}$ and $E_{t}\left(P_{t}>30 G e V\right.$ and $\left.E_{t}>30 G e V\right)$. Then, we looked the effect of radiation damage on $\mathrm{P}_{\mathrm{t}}, \mathrm{E}_{\mathrm{t}, \eta}$, and $\phi$. Here the important parameters are $\mathrm{P}_{\mathrm{t}}, \mathrm{E}_{\mathrm{t}}$, and $\eta$ because radiation damage is symmetric with $\phi$. Last, we looked jet tagging efficiencies both for two jets and one jet in HF, and we looked the effect of radiation damage on the efficiency for different regions of the HF calorimeter.

$$
\Delta \mathrm{R}=\sqrt{\Delta} \Delta \mathrm{\eta}^{2}+\Delta \varnothing^{2} \text { and } \Delta \mathrm{R}<0.3
$$

We have two forward jets reconstructed in the HF, and we applied a $P_{t}$ and $E_{t}$ cut of $30 \mathrm{GeV}$ on these jets and matched them to the generated jets. As can be seen in figures 
5.1 and 5.2, with increasing radiation damage there are some reconstructed jets at high $\mathrm{P}_{\mathrm{t}}$ and $E_{t}$ for small numbers of events. These events occur with jets in the overlap region between HE and HF. This effect is a suspected problem with the Fast Simulation reconstruction in the private version of CMSSW_4_2_3_SLHC4_patch1 from James Wetzel, January 202013.

Figure 5.3 shows $E_{t}, P_{t}, \eta$, and $\phi$ for $20 \mathrm{fb}^{-1}$ integrated luminosity for generated and reconstructed jets. As can be seen in figure 5.3, $\phi$ of both jets are not affected by radiation damage; differences in the graph are due to reconstruction and detector effects. The decrease in $E_{t}$ and $P_{t}$ between generated and reconstructed can be due to reconstruction effects in addition to radiation damage. Since the integrated luminosity is small, there is little impact from radiation damage, as seen in the $\eta$ distribution.

Figure 5.4 shows the overall jet tagging efficiency as a function of integrated luminosity. We calculated the overall jet tagging efficiency for VBF Higgs signal using formula (2). The efficiency is very small because we are looking the efficiency of two jets in the HF out of all the jets produced. Then, as can be seen in figure 5.5, we calculate the efficiency for single jets. We divided HF into four regions and calculated jet tagging efficiencies for each eta region using formula (3). We calculate the statistical error both for overall jet tagging efficiency and for the jet tagging efficiencies of the four different regions of the HF. The statistical error was calculated using formula (4). Table 5.1 and 5.2 show number of jets and efficiencies for each luminosity.

$$
\epsilon=\frac{\text { Number of events with two jets reconstructed, passing analysis cut, in HF }}{\text { Total events generated (500K) }(n)}
$$


Number of jets reconstructed, matching, passing analysis cuts

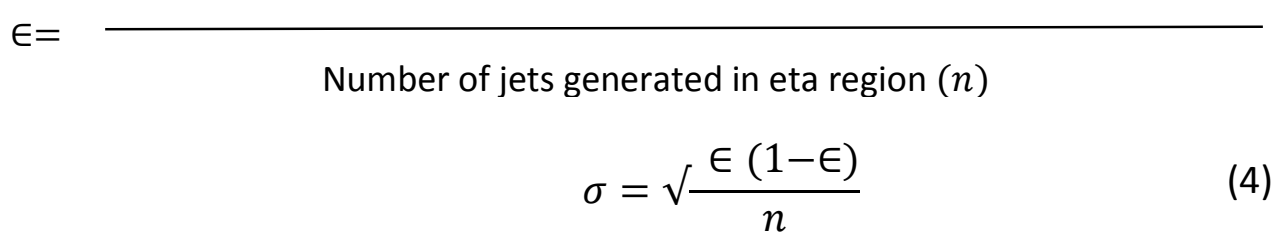

As can be seen in figure 5.4, radiation damage as expressed as integrated luminosity reduces the efficiency for tagging jets. In addition, as we mentioned in section 3.2, radiation damage has the most impact on the part that is closest to the collision beam. The closest region of the HF detector to the collision beam is $4.5<|\eta|<5.0$. That is why the efficiency of this region is the lowest compared to other regions of the detector. For the region $4.5<$ $|\eta|<5.0$ in figure 5.5, the efficiency starts approximately at $48 \%$ even though we do not have radiation damage in zero luminosity. This is because $\mathrm{P}_{t}$ and $\mathrm{E}_{t}$ of jets are lower in this region due to geometry. Figures 5.5 and 5.6 show how $\mathrm{P}_{t}$ and $\mathrm{E}_{t}$ of the jets change for four different regions of the $\mathrm{HF}$. 

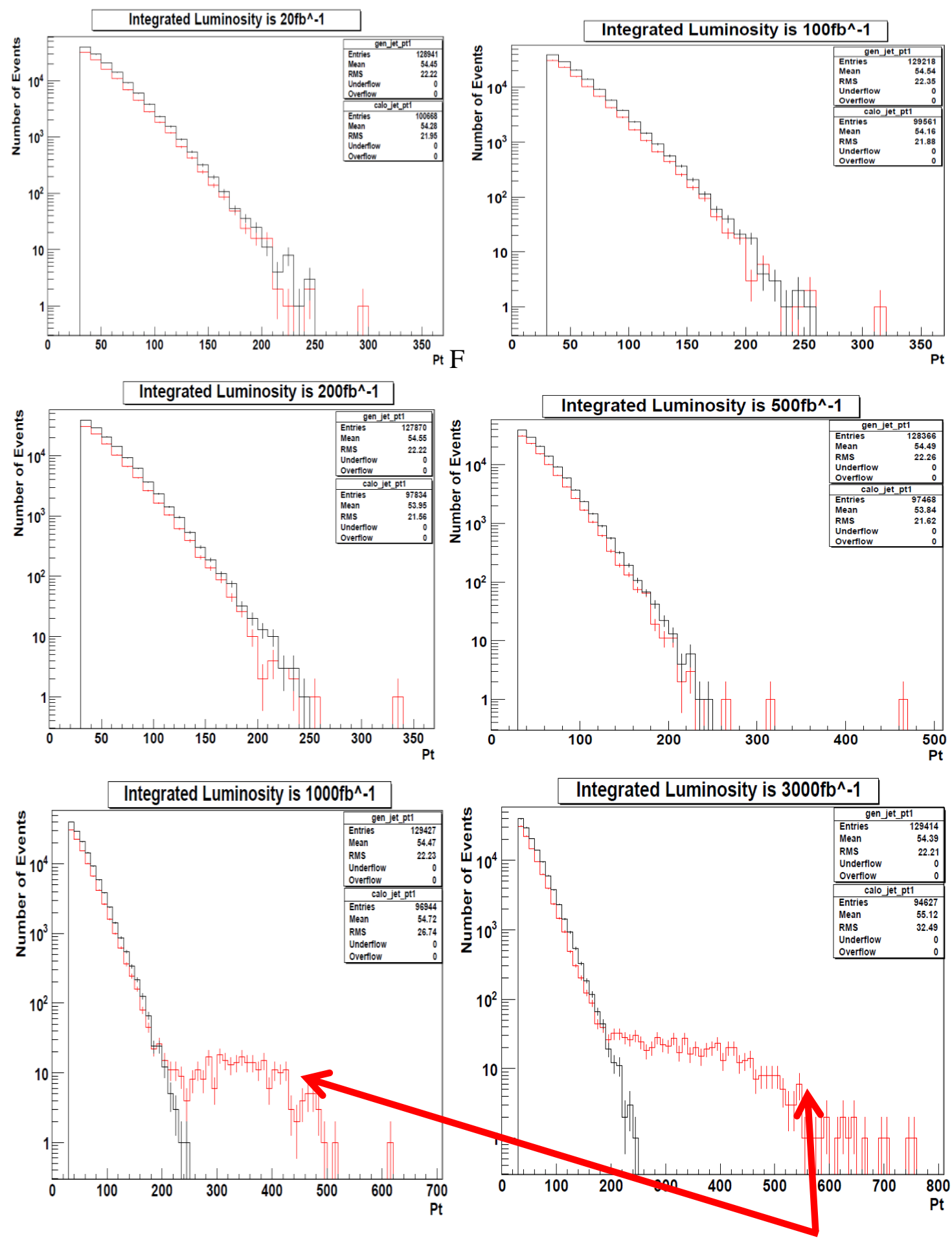

Possible FastSim reco issue in $\mathrm{HE} / \mathrm{HF}$ overlap region

Figure 5.1: Radiation damage effect on $P_{t}$ for $P_{t}>30 \mathrm{GeV}$. 


\section{Black - Gen Jet Red - Reco Jet}
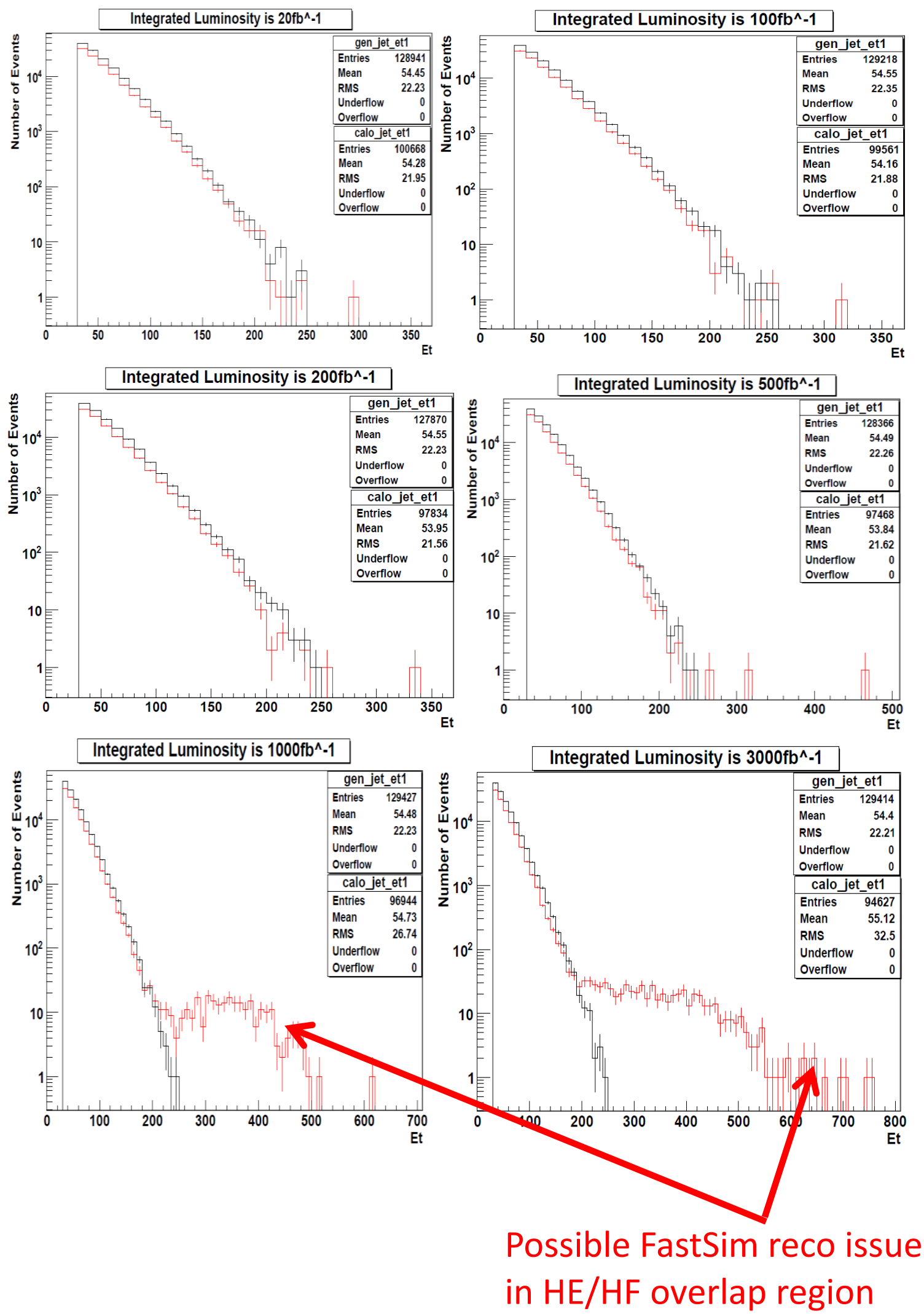

Figure 5.2: Radiation damage effect on $E_{t}$ for $E_{t}>30 \mathrm{GeV}$. 


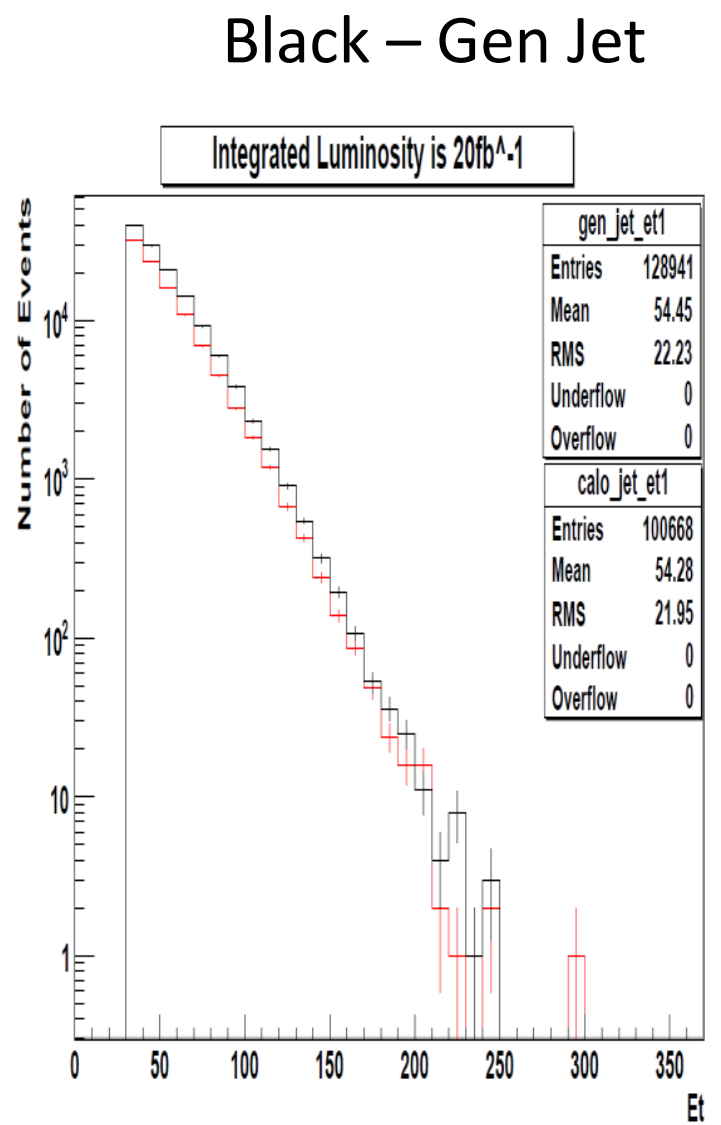

Red - Reco Jet

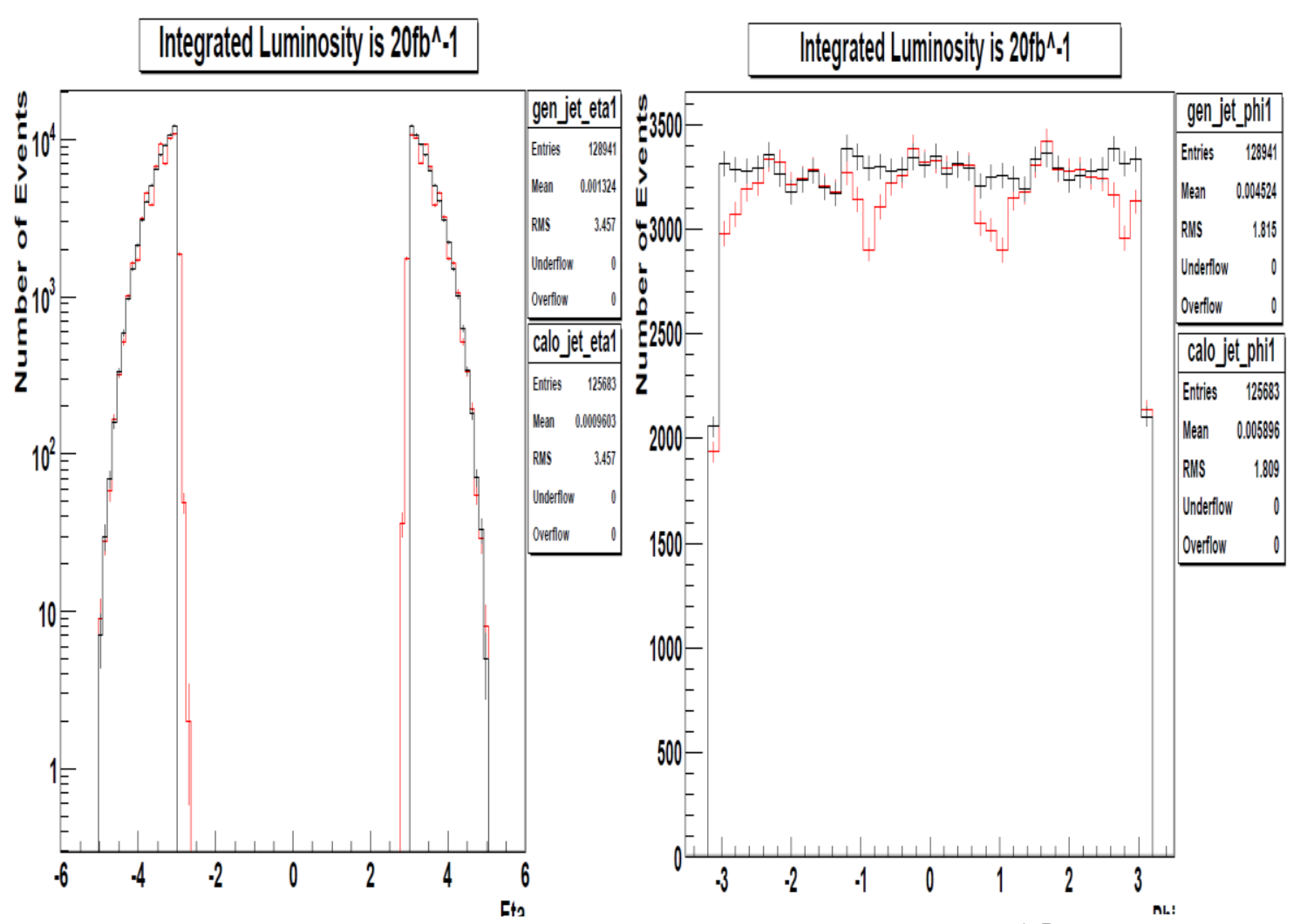

Figure 5.3: Effect of radiation damage on $E_{t}, P_{t}, \eta$, and $\phi$. 


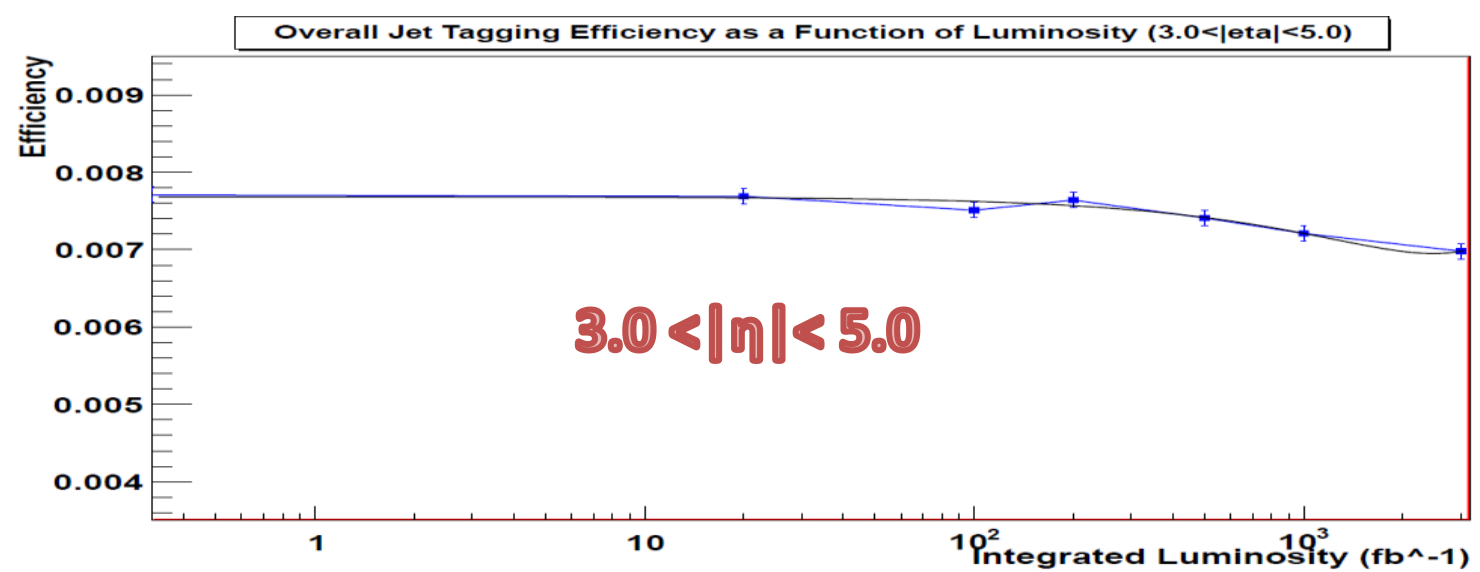

Figure 5.4: Overall jet tagging efficiency as a function of integrated luminosity for VBF Higgs Signal.

Table 5.1: Number of jets, overall efficiency and statistical errors for all luminosities.

\begin{tabular}{|c|c|c|c|c|c|c|c|c|}
\hline \multicolumn{2}{|l|}{ Luminosity } & \multirow{2}{*}{$\begin{array}{l}0 \\
0.00771\end{array}$} & \multirow{2}{*}{\begin{tabular}{|l|}
20 \\
0.00769
\end{tabular}} & \multirow{2}{*}{\begin{tabular}{|l|}
100 \\
0.00751 \\
\end{tabular}} & \multirow{2}{*}{$\begin{array}{l}200 \\
0.00764\end{array}$} & \multirow{2}{*}{$\begin{array}{l}500 \\
0.00741\end{array}$} & \multirow{2}{*}{$\begin{array}{l}1000 \\
0.00721\end{array}$} & \multirow{2}{*}{\begin{tabular}{|l|}
3000 \\
0.00698 \\
\end{tabular}} \\
\hline $\begin{array}{l}\text { Overall } \\
\text { Jet } \\
\text { Tagging } \\
\text { Efficiency }\end{array}$ & Eff & & & & & & & \\
\hline & $\begin{array}{l}\text { \# of } \\
\text { entries } \\
\text { for one } \\
\text { jet }\end{array}$ & 3857 & 3845 & 3753 & 3820 & 3704 & 3607 & 3490 \\
\hline & $\begin{array}{l}\text { Statistical } \\
\text { Error }\end{array}$ & 0.0001 & 0.0001 & 0.0001 & 0.0001 & 0.0001 & 0.0001 & 0.0001 \\
\hline
\end{tabular}


The relative decrease for overall efficiency $=\frac{0.00771-0.00698}{0.00771}=0.095$; efficiency loss is $9.5 \%$. As can be seen in figure 5.4, the overall signal efficiency decreases while the integrated luminosity increases.
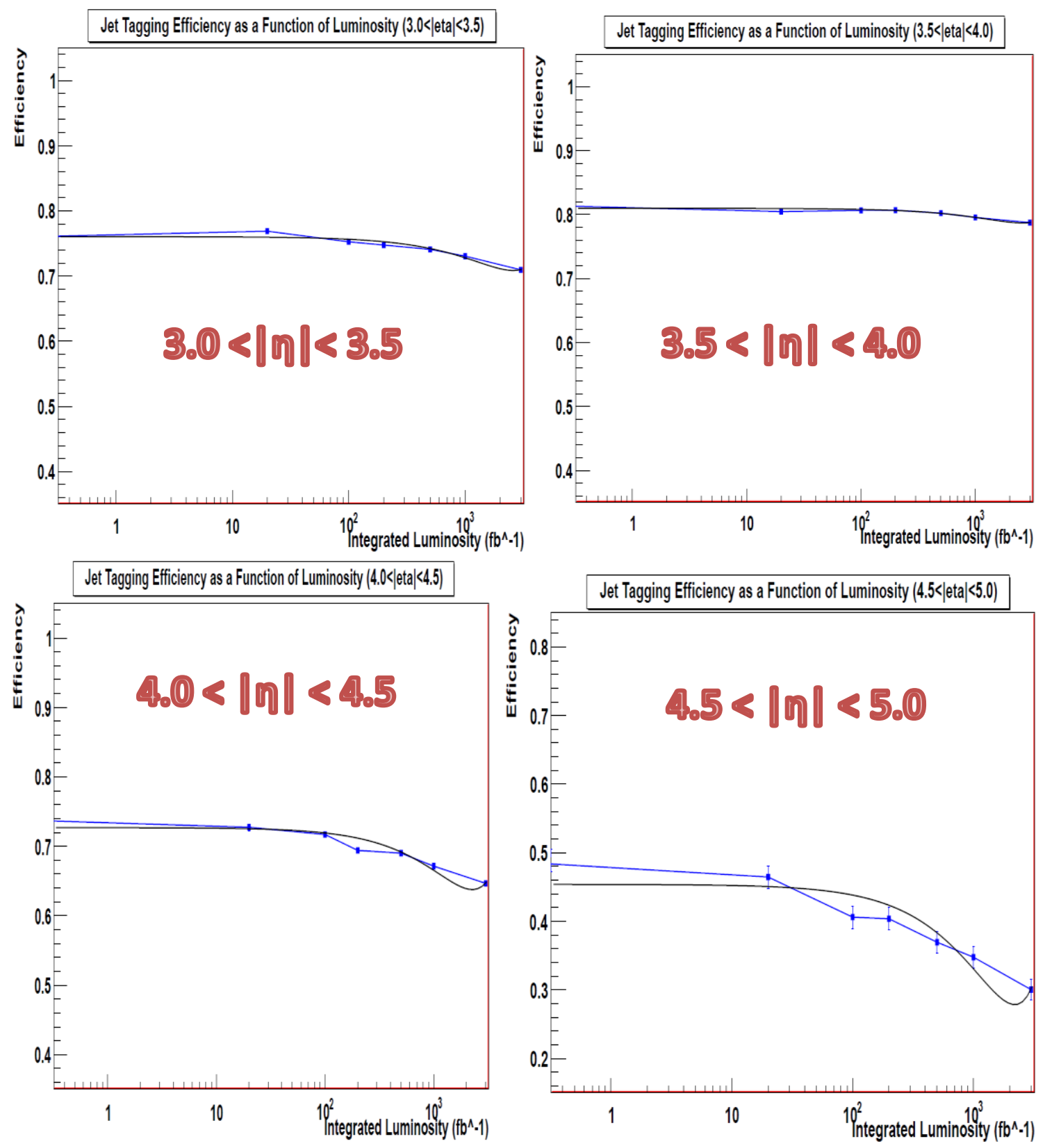

Figure 5.5: Jet tagging efficiency as a function of integrated luminosity for four regions of $\mathrm{HF}$ in $\eta$. 
As can be seen in figure 5.5, the loss in signal efficiency is most pronounced at lowest angles (closest to beam), but in this region the signal distribution is falling off. The analysis cut on Pt and Et has a larger effect at low angles due to geometry ( see figures 5.6 and 5.7).

Table 5.2: Number of jets, jet tagging efficiencies and statistical errors for all luminosities.

\begin{tabular}{|c|c|c|c|c|c|c|c|c|c|}
\hline \multicolumn{3}{|c|}{ Luminosity } & \multirow{2}{*}{$\begin{array}{l}0 \\
0.7590\end{array}$} & \multirow{2}{*}{$\begin{array}{l}20 \\
0.7688\end{array}$} & \multirow{2}{*}{$\begin{array}{l}100 \\
0.7525\end{array}$} & \multirow{2}{*}{$\begin{array}{l}200 \\
0.7475\end{array}$} & \multirow{2}{*}{$\begin{array}{l}500 \\
0.7409\end{array}$} & \multirow{2}{*}{$\begin{array}{l}1000 \\
0.7305\end{array}$} & \multirow{2}{*}{$\begin{array}{l}3000 \\
0.7095\end{array}$} \\
\hline & $3.0<|n|<3.5$ & Eff & & & & & & & \\
\hline \multirow{11}{*}{$\begin{array}{l}\text { Efficiency } \\
\text { for } \\
\text { different } \\
\text { regions } \\
\text { of HF }\end{array}$} & & $\begin{array}{l}\text { \# of } \\
\text { entries } \\
\text { for one } \\
\text { jet }\end{array}$ & 63629 & 64689 & 63233 & 62289 & 62747 & 61753 & 59563 \\
\hline & & $\begin{array}{l}\text { Statistical } \\
\text { Error }\end{array}$ & 0.0015 & 0.0015 & 0.0015 & 0.0015 & 0.0015 & 0.0015 & 0.0015 \\
\hline & $3.5<|n|<4.0$ & Eff & 0.8154 & 0.8047 & 0.8066 & 0.8069 & 0.8023 & 0.7956 & 0.7875 \\
\hline & & $\begin{array}{l}\text { \# of } \\
\text { entries } \\
\text { for one } \\
\text { jet }\end{array}$ & 32056 & 31217 & 31633 & 31030 & 31200 & 30756 & 30816 \\
\hline & & $\begin{array}{l}\text { Statistical } \\
\text { Error }\end{array}$ & 0.0020 & 0.0020 & 0.0020 & 0.0020 & 0.0020 & 0.0020 & 0.0020 \\
\hline & $4.0<|\eta|<4.5$ & Eff & 0.7389 & 0.7274 & 0.7170 & 0.6941 & 0.6901 & 0.6714 & 0.6467 \\
\hline & & $\begin{array}{l}\text { \# of } \\
\text { entries } \\
\text { for one } \\
\text { jet }\end{array}$ & 7563 & 7379 & 7237 & 7131 & 7012 & 6966 & 6647 \\
\hline & & $\begin{array}{l}\text { Statistical } \\
\text { Error }\end{array}$ & 0.0043 & 0.0044 & 0.0045 & 0.0045 & 0.0046 & 0.0046 & 0.0047 \\
\hline & $4.5<|n|<5.0$ & Eff & 0.4889 & 0.4646 & 0.4061 & 0.4037 & 0.3695 & 0.3477 & 0.3004 \\
\hline & & $\begin{array}{l}\text { \# of } \\
\text { entries } \\
\text { for one } \\
\text { jet }\end{array}$ & 463 & 433 & 370 & 369 & 351 & 331 & 304 \\
\hline & & $\begin{array}{l}\text { Statistical } \\
\text { Error }\end{array}$ & 0.0162 & 0.0163 & 0.0163 & 0.0162 & 0.0157 & 0.0154 & 0.0144 \\
\hline
\end{tabular}

The relative decrease in efficiency for different regions of the HF detector has been calculated. For the region $3<|\eta|<3.5$, the relative decrease $=\frac{0.7590-0.7095}{0.7590}=0.066$; the 
efficiency loss is $6.6 \%$. For the region $3.5<|\eta|<4.0$, the relative decrease $=\frac{0.8154-0.7875}{0.8154}=$ 0.034 ; the efficiency loss is $3.4 \%$. For the region $4.0<|\eta|<4.5$, the relative decrease $=\frac{0.7389-0.6467}{0.7389}=0.124$; the efficiency loss is $12.4 \%$. For the region $4.5<|\eta|<5.0$, the relative decrease $=\frac{0.4889-0.3004}{0.4889}=0.386 ;$ the efficiency loss is $38.6 \%$.

We apply the VBF Higgs analysis cuts on the transverse momentum and energy of the jets, leading to a reduction of efficiency in the detector. The requirement for VBF Higgs signal is $30 \mathrm{Gev}$ transverse momentum cut. In this research, $30 \mathrm{GeV}$ transverse energy cut was also used. Although the change in $\mathrm{P}_{\mathrm{t}}$ and $\mathrm{E}_{\mathrm{t}}$ is related to the efficiency, the radiation damage and the efficiency are inversely proportional.

As can be seen in table 5.2, the efficiency loss increases from the region $3.0<|\eta|<$ 3.5 to the region $4.5<|\eta|<5.0$. Both table 5.2 and figure 5.5 show that the signal efficiency decreases while integrated luminosity increases. For the region $3.0<|\eta|<3.5$ in figure 5.5, there is an overlap between the HF and $\mathrm{HE}$ at $\eta$ of 3.0 which may be cause of the reduction of efficiency. The region $4.5<|\eta|<5.0$ starts at $48 \%$ efficiency in $0 \mathrm{fb}^{-1}$ integrated luminosity. As can be seen in the figure 5.6 and 5.7, this is because of geometry since the analysis uses transverse variables, jets with the same energy deposited in the detector will be reconstructed with a lower transverse energy at lower angles with respect to the beam. 




Red - Reco Jet
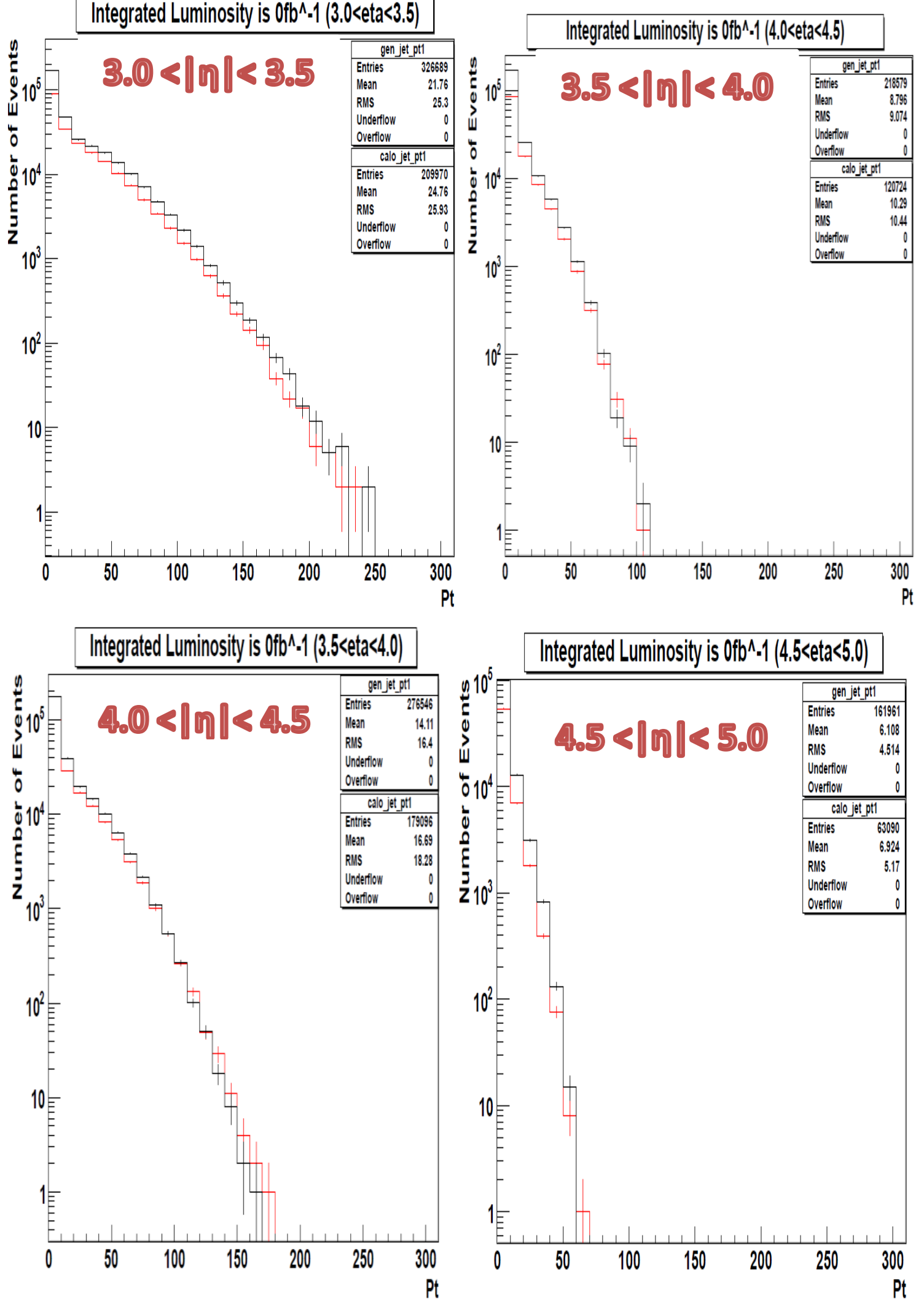

Figure 5.6: $\mathrm{P}_{t}$ distribution for four different regions of HF shown for no radiation damage. 
Black - Gen Jet Red - Reco Jet
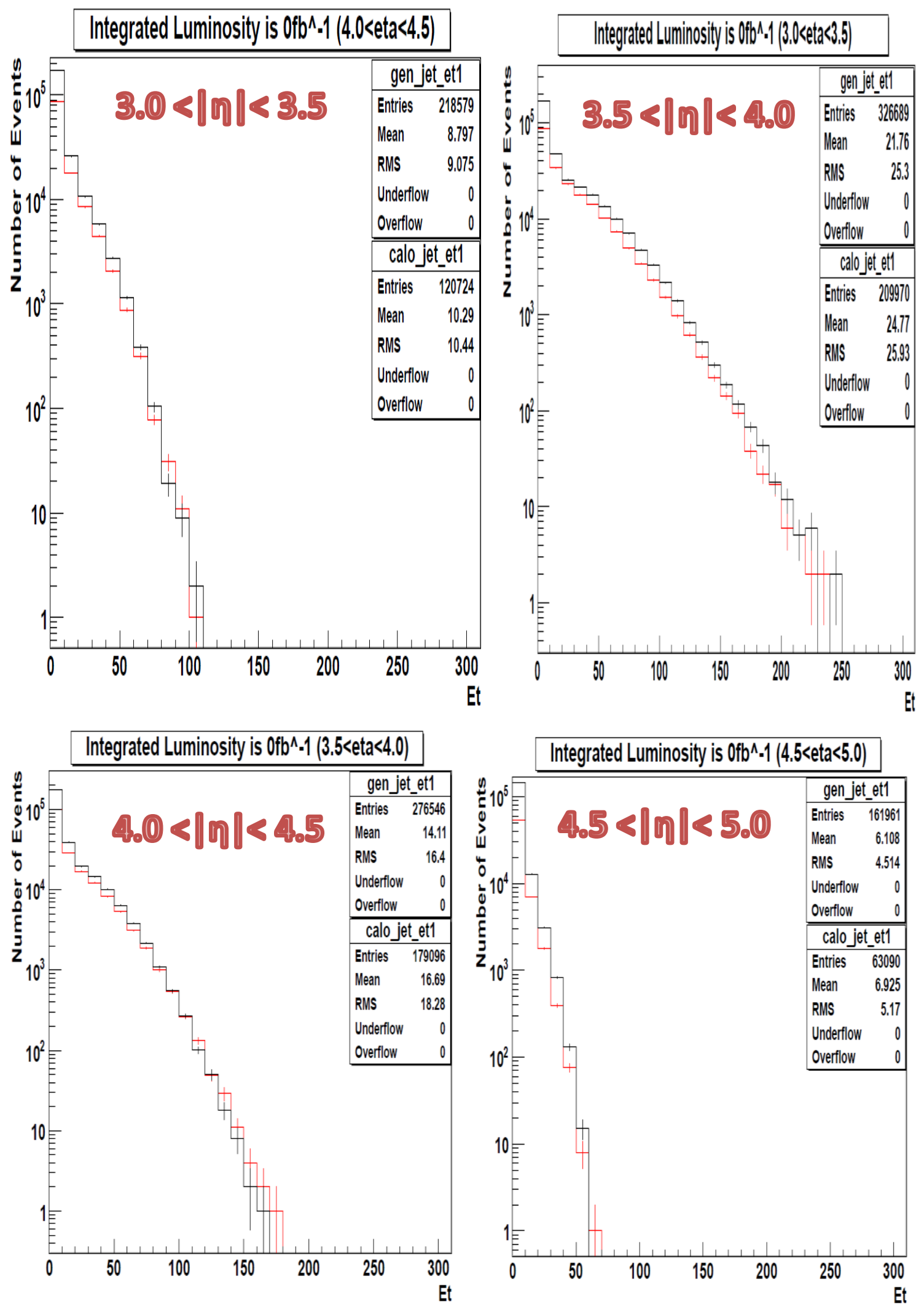

Figure 5.7: $\mathrm{E}_{\mathrm{t}}$ distribution for four different regions of $\mathrm{HF}$ shown for no radiation damage. 


\section{CHAPTER 6}

\section{SUMMARY AND CONCLUSION}

The LHC is the biggest particle physics accelerator that is used to solve particle physics questions, and the CMS is one of the main detectors at the LHC. There are four Hadronic Calorimeters in the CMS, and the Hadronic Forward Calorimeter is one of them. Physicists collide two beams of protons at the LHC and the collisions cause a high radiation environment. Thus, the detectors at the LHC are affected by radiation. VBF production is one of the methods that has being used to search for the Higgs boson. For our study, we looked at how the VBF signal in the HF detector changes with radiation damage.

In this analysis, jets from the VBF Higgs signal are selected using a threshold (30 $\mathrm{GeV})$ on the transverse momentum $\left(\mathrm{P}_{\mathrm{t}}\right)$ and transverse energy $\left(\mathrm{E}_{\mathrm{t}}\right)$ of the jets. The effect of radiation damage is observed both for the total signal and for four different regions of the $\mathrm{HF}$ calorimeter. The results showed that the regions which are closest to the collision point were affected more compared to the other regions. This result agrees with the expected one. The effect of radiation damage was observed on the VBF Higgs signal and we saw that the VBF signal was not significantly decreased by radiation. In addition, the relationship between $\mathrm{P}_{t}, \mathrm{E}_{\mathrm{t}}$ and the efficiency were observed.

All these observations were made without applying a correction factor to restore the attenuated energy to expected levels. This would normally be done as a calibration step. For the next step of this project, we will apply the corrections and observe the results. For future work, it is possible to look at the physics impact on other signatures where HF plays a major role, such as missing $E_{t}$ based searches. 


\section{BIBLIOGRAPHY}

[1] D. Griffiths, Introduction to Elementary Particles, Wiley-VCH, 2009.

[2] D0 Collaboration, "Search for Neutral Higgs Bosons Decaying to Tau Pairs in ppbar Collisions at sqrt(s) $=1.96 \mathrm{TeV}, "$ Physical Review Letter, vol. 97, no. 12, pp. 121802-121809, 2006.

[3] CERN, "New results indicate that new particle is a Higgs boson," CERN Accelerating Science, 14 March 2013. [Online]. Available: http://home.web.cern.ch/about/updates/2013/03/new-results-indicate-newparticle-higgs-boson.

[4] D. Green, "Vector Boson Fusion and Quadratic Boson Couplings," 2003. [Online]. Available: http://arxiv.org/abs/hep-ph/0306160

[5] D. J. Mangeol, "Physics with tau's in the final states at CMS," CMS CR081, 2006.

[6] C. Foudas, A. Nikitenko, M. Takahashi, "Observation of the Standard Model Higgs boson $\mathrm{H} \rightarrow \tau \tau \rightarrow$ lepton+jet Channel," CMS Note, vol. 088, 2006.

[7] Yajun Mao, et all, "Mass Reconstruction Methods for $\mathrm{qqH}, \mathrm{H} \rightarrow \tau \tau \rightarrow$ Leptonic Decay," CMS AN -2009/187, 2009.

[8] CERN, "LHC Machine," JINST 3 S08001, 2008.

[9] L. Benucci, "Searching for extra-dimensions at CMS," Journal of Physics, vol. $171,2009$.

[10] Whitney Clavin, J.D. Harrington, " Planck Mission Brings Universe Into Sharp Focus," 2013. [Online]. Available:

http://www.nasa.gov/mission_pages/planck/news/planck20130321.html. [Accessed 2606 2013].

[11] CMS Collaboration, "The CMS experiment at the CERN LHC," JINST 3 S08004, 2008.

[12] CMS Collaboration, Detector Performance and Software -Physics Technical Design Report, CERN/LHCC 2006-001, 2006. 
[13] A.Penzo, Y. Onel (for the CMS collaboration), "The CMS-HF quartz fiber calorimeters," Journal of Physics, vol. 160, 2009.

[14] P. C. Bhat, N. V. Mokhov, A. P. Singh and S. B. Beri, "Simulation Studies of the Radiation Environment in the CMS Detector for pp Collisions at the LHC," CMS NOTE -2013/001, 2013.

[15] M. Huhtinen, The Radiation Environment at the CMS Experiment at the LHC, CH -1211 Geneva, Switzerland: CERN, 1996.

[16] B.Bilki, V.Khristenko, J.Nachtman, Y.Onel, J.Wetzel, D.Winn, T.Yetkin, "Studies for the Phase II HF Upgrade," CMS Internal Note, 2013.

[17] K. Cankocak, N. M Bakirci, S. Cerci, E. Gulmez, J. P. Merlo, Y. Onel, F. Ozok, I. Schmidt, N. Sonmez, "Radiation-hardness measurements of high OH content quartz fibers irradiated with $24 \mathrm{GeV}$ protons up to $1.25 \mathrm{Grad}$," CMS Note, 2007.

[18] A. Rao, "New CMS results at Moriond (Electroweak)," 2013. [Online]. Available: http://cms.web.cern.ch/news/new-cms-results-moriond-electroweak-2013.

[19] V. Ilisie, "S.M. Higgs Decay and Production Channels," M.S Thesis, University of Valencia, Valencia, 2010-2011. [Online]. Available: http://ific.uv.es//hcpheno/PhDthesis/master_vilisie.pdf.

[20] Monica Luisa Vazquez Acosta (on behalf of the CMS Collaborations), "Vector Boson Fusion Production of the Standard Model Higgs at the LHC," in Proceedings of Science, 2008.

[21] D. Green, "Vector Boson Fusion and Quadratic Boson Couplings," 2003. [Online]. Available: http://arxiv.org/abs/hep-ph/0306160. 